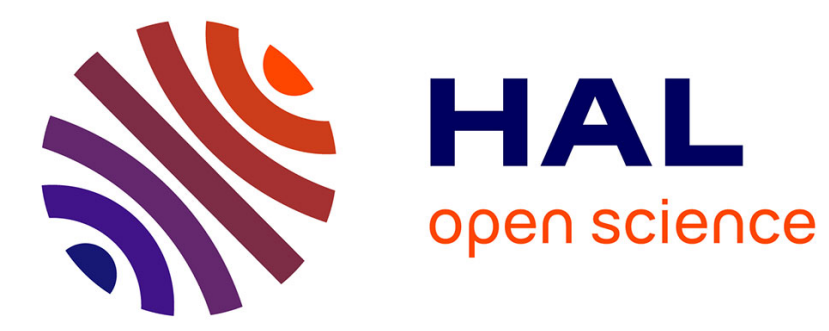

\title{
Local regularity for texture segmentation: Combining wavelet leaders and proximal minimization
}

Nelly Pustelnik, Herwig Wendt, Patrice Abry

\section{To cite this version:}

Nelly Pustelnik, Herwig Wendt, Patrice Abry. Local regularity for texture segmentation: Combining wavelet leaders and proximal minimization. IEEE International Conference on Acoustics, Speech, and Signal Processing (ICASSP), May 2013, Vancouver, Canada. pp.x+4. hal-00826839v1

\section{HAL Id: hal-00826839 \\ https://hal.science/hal-00826839v1}

Submitted on 28 May 2013 (v1), last revised 4 May 2015 (v2)

HAL is a multi-disciplinary open access archive for the deposit and dissemination of scientific research documents, whether they are published or not. The documents may come from teaching and research institutions in France or abroad, or from public or private research centers.
L'archive ouverte pluridisciplinaire HAL, est destinée au dépôt et à la diffusion de documents scientifiques de niveau recherche, publiés ou non, émanant des établissements d'enseignement et de recherche français ou étrangers, des laboratoires publics ou privés. 


\title{
LOCAL REGULARITY FOR TEXTURE SEGMENTATION: COMBINING WAVELET LEADERS AND PROXIMAL MINIMIZATION
}

\author{
Nelly Pustelnik ${ }^{1}$, Herwig Wendt ${ }^{2}$, Patrice Abry ${ }^{1}$ \\ ${ }^{1}$ Physics Dept. - ENSL, UMR CNRS 5672, F-69364 Lyon, France, firstname. lastnamedens-lyon. fr \\ ${ }^{2}$ IRIT - ENSEEIHT, UMR CNRS 5505, F-31062 Toulouse, France, herwig • wendt @irit. fr
}

\begin{abstract}
Texture segmentation constitutes a classical yet crucial task in image processing. In many applications of very different natures (biomedical, geophysics,...) textures are naturally defined in terms of their local regularity fluctuations, which can be quantified as the variations of local Hölder exponents. Furthermore, such images are often naturally embedded in the class of piece-wise constant local regularity functions. The present contribution aims at proposing and assessing a segmentation procedure for this class of images. Its originality is twofold: First, local regularity is estimated using wavelet leaders, a novel multiresolution quantity recently introduced for multifractal analysis but barely used in local regularity measurement, comparisons against wavelet coefficient based estimation are conducted; Second, the challenging minimal partition problem underlying segmentation is convexified and conducted within a customized proximal framework. The estimation of the number of regions and their target regularity is obtained from a total-variation estimate that enables the actual use of proximal minimization for texture segmentation. Performance is assessed and illustrated on synthetic textures.
\end{abstract}

Index Terms - Texture segmentation, piece-wise constant, local regularity, wavelet leaders, proximal minimization.

\section{MOTIVATION, RELATED WORKS, CONTRIBUTIONS}

Texture segmentation from local regularity fluctuations. Texture characterization and segmentation constitutes a challenging task in image processing (see e.g., $[1,2,3,4,5]$ and references therein). In a variety of applications of possibly very different natures, the relevant information characterizing textures, and thus conveying the information to be analyzed, consists of the fluctuations across space of their local regularity. This is notably the case for biomedical textures such as bone [6] or breast [7] (see also [8] for a review), for surface imagery [9], for satellite imagery [10], or for image registration $[11,12]$.

Often, local regularity is assessed in terms of the so-called Hölder exponent $h(\underline{x})$ [13]. In essence, $h(\underline{x})$ consists of the power law exponent quantifying the decrease of the powers of local fluctuations, measured via multiresolution quantities $T_{X}(a, \underline{x})$ (e.g., wavelet coefficients) around a given space position $\underline{x}$ when the analysis scales $a$ goes towards fine scales, i.e., $\mathbb{E} T_{X}(a, \underline{x})^{2} \simeq$ $C(\underline{x}) a^{2 h(\underline{x})}$ when $a \rightarrow 0$.

Related works: Hölder exponent estimation and segmentation. Local regularity based analysis of textures can naturally be organized into two sub-questions: i) Accurately estimating the Hölder exponents for each location; ii) Splitting the image into regions where estimates can be considered constant, so as to achieve texture segmentation. Though these issues have received significant efforts over the past two decades for 1D signals (cf. e.g., [14, 15]), much less contributions were dedicated to 2D fields or images (see a contrario [12] and references therein).

Estimation relies on two key choices, with crucial impact on performance: Multiresolution quantities $T_{X}(a, \underline{x})$ from which the $h(\underline{x})$ are measured; Range of analysis scales $a$ to be practically involved in the estimation. For multiresolution quantities, most works conducted so far relied on either increments, oscillations or wavelet coefficients. Bilinear time-scale representations were also used [15]. The choice of the range of scales is naturally framed into a classical bias-variance trade-off. While theoretically, estimation should be conducted in the limit of fine scales, practically a finite range of scales $a_{m} \leq a \leq a_{M}$ has to be used. Choosing a large range of scales yields smaller estimation variance at the price of increasing the bias because the $T_{X}(a, \underline{x})$ at large scales are less well localized (cf. e.g., $[16,15])$.

For segmentation, i.e., to detect changes amongst Hölder exponents measured at different locations, this trade-off also turns crucial. This is why both estimation and change detection have mostly been conducted in model dependent frameworks. Fractional Brownian motion (fBm) [17], the paradigm model for scale invariance, implies a constant and unique Hölder exponent across sample fields. Multifractional Brownian motion extends $\mathrm{fBm}$ by allowing piece-wise smooth variations of $h(\underline{x})$ across space (while preserving joint Gaussianity) [18] and constitutes essentially the only model with spacedependent $h$ that has been studied and used. The problem has mostly been addressed for 1D signals and barely for images (see a contrario $[12,7]$ which aimed at identifying particular points in the image defined by specific values of $h(\underline{x})$ ). Furthermore, to our knowledge, little work has been published aiming at detecting changes in $h(\underline{x})$ in a context where an exact model is not a priori assumed (see a contrario [15] for 1D signals).

With respect to the generic issue of image segmentation, efficient variational methods were proposed [19, 20, 21, 22, 23, 24], however none of them guarantees convergence towards a global minimum, and thus, obtained solutions strongly depend on initialization. Instead, a convex relaxation of the minimal partition techniques were proposed in [25, 26]. In [26], the algorithmic solution is based on a Arrow-Hurwicz type primal-dual algorithm but requires inner iterations and upper boundedness of the primal energy in order to improve convergence speed. Also, a proximal solution in a context of disparity estimation was proposed in [27]. Alternatively, recent techniques are framed into variational principles, making use of comparisons between pairs of neighboring patches $[28,3]$. Notably, in [28], a convex relaxation of the original problem was envisaged so as to ensure to find the global minimizer, while in [3] a more general but non-convex framework was proposed, which yields good results at the price of a well-chosen initialization. However, the computational cost is a limitation of these methods. 
Goals and contributions. In this context, the first originality of the present contribution consists in performing region-segmentation of images, that excludes the recourse to a fully parametric model for the data, but instead only assumes that images belong to the class of piecewise constant Hölder exponent functions. Its second originality lies in a twofold departure from earlier works: For multiresolution quantities, wavelet leaders are chosen and shown to yield improved statistical performance for Hölder exponent estimation, compared to the classical wavelet coefficients (cf. Section 2); For segmentation, using estimated Hölder exponents as inputs, a proximal algorithm is devised. It essentially relies on the customization of the formulation proposed in [27], which expands on that proposed in [26] to efficiently handle the resulting large size problem. Further, we propose an original method to a priori estimate the number of regions as well as their mean regularity, an initialization step that turns compulsory and crucial for the actual use of the proximal approach in that context. These are detailed in Section 3.

This combination (use of wavelet leaders and of proximal algorithm with original initialization) enables us to propose an efficient data-model free piecewise constant Hölder exponent minimal partition image segmentation procedure, that furthermore depends on very few parameters that need to be tuned manually.

\section{WAVELET COEFFICIENTS AND LEADERS FOR HÖLDER EXPONENT ESTIMATION}

Local regularity and Hölder exponent. Let $X$ denote the bounded 2D function (image) to be analyzed. Local regularity around position $\underline{x}_{0} \in \mathbb{R}^{2}$ is measured by the so-called Hölder exponent $h\left(\underline{x}_{0}\right)$, defined as the largest $\alpha>0$, such that there exists a constant $C>0$ and a polynomial $P_{\underline{x}_{0}}$ of degree less than $\alpha$, such that $\left|X(\underline{x})-P_{\underline{x}_{0}}(\underline{x})\right| \leq C\left|\underline{x}-\underline{x}_{0}\right|^{\alpha}$ in a neighborhood $\underline{x}$ of $\underline{x}_{0}$. When $h\left(\underline{x}_{0}\right)$ is close to 0 , the image is locally very irregular and close to discontinuous. Conversely, a large $h\left(\underline{x}_{0}\right)$ corresponds to a locally smooth field. For example, when $h\left(\underline{x}_{0}\right)$ increases towards 2 then the fields becomes smoother and smoother up to being differentiable. Fig. 1-b below displays a Gaussian texture, with piecewise constant Hölder exponents, the region with the lowest $h$ (outer ring) is more irregular then the one with the largest $h$ (inner ring).

Wavelet coefficients. Let $\phi(x)$ and $\psi(x)$ denote the scaling function and mother wavelet defining a 1D multiresolution analysis. Let the 2D wavelets be defined as: $\psi^{(0)}(\underline{x})=\phi\left(x_{1}\right) \phi\left(x_{2}\right), \psi^{(1)}(\underline{x})=$ $\psi\left(x_{1}\right) \phi\left(x_{2}\right), \psi^{(2)}(\underline{x})=\phi\left(x_{1}\right) \psi\left(x_{2}\right), \psi^{(3)}(\underline{x})=\psi\left(x_{1}\right) \psi\left(x_{2}\right)$ with $\underline{x}=\left(x_{1}, x_{2}\right)$. The collections $\psi_{j, \underline{k}}^{(m)}(\underline{x})=2^{-j} \psi^{(m)}\left(2^{-j} \underline{x}-\underline{k}\right)$ of dilated (to scales $a=2^{j}$ ) and translated (to space positions $\underline{x}=2^{j} \underline{k}$ ) templates of $\psi_{0}$ form a basis of $L^{2}\left(\mathbb{R}^{2}\right)$ for well chosen functions $\psi$. Let $d_{X}^{(m)}(j, \underline{k})=\left\langle X, \psi_{j, \underline{k}}^{(m)}\right\rangle$ denote the $\left(L^{1}\right.$ normalized) coefficients of $X$ of the so-called discrete wavelet transform (DWT) of $X$. Readers may refer to [29] for further details.

Wavelet leaders. Wavelet leaders were recently introduced in the context of multifractal analysis $[13,30]$, their use in the context of local regularity measurement has however been barely considered. The wavelet leader $L_{X}(j, \underline{k})$, located around position $\underline{x}_{0}=2^{j} \underline{k}$, is defined as the local supremum of all wavelet coefficients taken within a spatial neighborhood across all finer scales $2^{j^{\prime}} \leq 2^{j}$ :

$$
L_{X}(j, \underline{k})=\sup _{\substack{m=1,2,3, \lambda_{j^{\prime}, \underline{k}^{\prime}} \subset \Lambda_{j, \underline{k}}}}\left|d_{X}^{(m)}\left(j^{\prime}, \underline{k}^{\prime}\right)\right|,
$$

$\lambda_{j, \underline{k}}=\left[\underline{k} 2^{j},(\underline{k}+1) 2^{j}\right), \Lambda_{j, \underline{k}}=\bigcup_{p \in\{-1,0,1\}^{2}} \lambda_{j, \underline{k}+p}[13,30]$.

Local regularity estimation. It has long been known that, for large (but not all) classes of images [13], wavelet coefficients reproduce the Hölder exponent $h\left(\underline{x}_{0}\right)$, in so far as, for all $\underline{k}_{0}$ such that $2^{j} \underline{k}_{0} \approx \underline{x}_{0}: \frac{1}{3} \sum_{m=1}^{3}\left|d_{X}^{(m)}\left(j, \underline{k}_{0}\right)\right| \simeq C_{d}\left(\underline{x}_{0}\right) 2^{j h\left(\underline{x}_{0}\right)}$ when $2^{j} \rightarrow 0$. More recently, it has been shown $[13,30]$ that wavelet leaders systematically (that is: for all classes of images) reproduce the Hölder exponent $h\left(\underline{x}_{0}\right): L_{X}\left(j, \underline{k}_{0}\right) \simeq C_{L}\left(\underline{x}_{0}\right) 2^{j h\left(\underline{x}_{0}\right)}$ when $2^{j} \rightarrow 0$. This naturally leads to the estimation of $h\left(\underline{x}_{0}\right)$ by means of linear regressions:

$$
\begin{aligned}
& \widehat{h}_{d}\left(\underline{x}_{0}\right)=\sum_{j=j_{1}}^{j_{2}} w_{j} \ln \frac{1}{3} \sum_{m=1}^{3}\left|d_{X}^{(m)}\left(j, \underline{k}_{0}\right)\right|, \\
& \widehat{h}_{L}\left(\underline{x}_{0}\right)=\sum_{j=j_{1}}^{j_{2}} w_{j} \ln L_{X}\left(j, \underline{k}_{0}\right),
\end{aligned}
$$

with weights $w_{j}$ chosen to perform non weighted regression [15]. Section 4 compares estimates obtained from either wavelet coefficients or leaders and shows that the latter yield a substantial decrease in variance and are thus to be preferred.

\section{MINIMAL PARTITION SEGMENTATION USING PROXIMAL TOOLS}

Let $\Omega \subset \mathbb{R}^{2}$ denote a spatially continuous domain and $f \in L^{2}(\Omega)$ the image that we intend to label. Note that, in Section $4, f$ will successively model the estimated Hölder exponents obtained from wavelet coefficients or leaders, i.e., $f=\widehat{h}_{d}$ or $f=\widehat{h}_{L}$. The Mumford-Shah approach in [20] consists in computing a piecewise constant approximation of data $f$. Assuming that $f$ can be approximated by $Q$ labels such that $\left(\Omega_{q}\right)_{1 \leq q \leq Q}$ models the $Q$ distinct regions, this problem can be written as

$$
\begin{aligned}
& \min _{\Omega_{1}, \ldots, \Omega_{Q}} \sum_{q=1}^{Q} \int_{\Omega_{q}}\left(f-v_{q}\right)^{2} d x+\frac{1}{2} \sum_{q=1}^{Q} \operatorname{Per}\left(\Omega_{q}\right) \\
& \text { s.t. } \quad\left\{\begin{array}{l}
\bigcup_{q=1}^{Q} \Omega_{q}=\Omega, \\
(\forall q \neq p), \Omega_{q} \cap \Omega_{p}=\emptyset,
\end{array}\right.
\end{aligned}
$$

where $v_{q}$ stands for the value of $f$ in region $\Omega_{q}$ (with, by convention, $v_{q} \leq v_{q+1}$ ), where the left-hand-side term consists of the sum of the variances of $f$ over all regions, and where $\operatorname{Per}\left(\Omega_{q}\right)$ measures the perimeter of region $\Omega_{q}$, and where the constraints imposed on $\Omega_{q}$ ensure to obtain a non-overlapping partition of the image. The discrete analogue of Model (4) is the Potts model which is known to be NP-hard to solve. A solution to circumvent this difficulty consists in relaxing this non convex formulation into a convex approximation [26].

From now on, the sampled version of the image $f$ is considered and denoted as a vector of pixels $f=\left(f^{(n)}\right)_{1 \leq n \leq N} \in \mathbb{R}^{N}$. The $Q$ regions $\Omega_{q}$ are labeled through an auxiliary variable $u=$ $\left(u^{(n)}\right)_{1 \leq n \leq N}$, such that $u^{(n)}=v_{q}$ if and only if the pixel $n$ belongs to the region $\Omega_{q}$. Further, one can introduce $Q$ binary functions $\theta=\left(\theta_{1}, \ldots, \theta_{Q}\right)$ such that, for every $q \in\{1, \ldots, Q\}$,

$$
(\forall n \in\{1, \ldots, N\}), \quad \theta_{q}^{(n)}= \begin{cases}1 & \text { if } u^{(n)} \geq v_{q}, \\ 0 & \text { otherwise. }\end{cases}
$$

It results that the labeling function $u$ can be recovered from $\theta=$ $\left(\theta_{1}, \ldots, \theta_{Q}\right)$ through the relation

$$
(\forall n \in\{1, \ldots, N\}), \quad u^{(n)}=\sum_{q=1}^{Q}\left(v_{q}-v_{q-1}\right) \theta_{q}^{(n)},
$$


with $v_{0}=0$. A bijection between $u$ and $\theta$ is guaranteed by constraining, for every $n \in\{1, \ldots, N\}$ :

$\mathcal{B}_{n}=\left\{\theta^{(n)} \in\{0,1\} \times \ldots \times\{0,1\}, 1 \geq \theta_{1}^{(n)} \geq \ldots \geq \theta_{Q}^{(n)} \geq 0\right\}$,

whose convex relaxation is

$\overline{\mathcal{B}}_{n}=\left\{\theta^{(n)} \in[0,1] \times \ldots \times[0,1], 1 \geq \theta_{1}^{(n)} \geq \ldots \geq \theta_{Q}^{(n)} \geq 0\right\}$.

Moreover, for every $n \in\{1, \ldots, N\}, \theta_{1}^{(n)}=1$.

Convex criterion The convexification of Model (4) leads to the following minimization problem involving the $Q$ binary functions $\theta=\left(\theta_{1}, \ldots, \theta_{Q}\right)[26]$ :

$$
\begin{aligned}
& \underset{\theta_{1}, \ldots, \theta_{Q}}{\operatorname{minimize}} \sum_{q=1}^{Q-1} \sum_{n=1}^{N}\left(\theta_{q}^{(n)}-\theta_{q+1}^{(n)}\right)\left(f^{(n)}-v_{q}\right)^{2} \\
&+\sum_{n=1}^{N} \theta_{Q}^{(n)}\left(f^{(n)}-v_{Q}\right)^{2}+\frac{1}{2} \sum_{q=1}^{Q} \rho_{\mathrm{TV}}\left(H \theta_{q}, V \theta_{q}\right) \\
& \text { s.t. } \quad\left\{\begin{array}{l}
(\forall n \in\{1, \ldots, N\}), \theta_{1}^{(n)}=1, \\
(\forall n \in\{1, \ldots, N\}), 1 \geq \theta_{2}^{(n)} \geq \ldots \geq \theta_{Q}^{(n)} \geq 0,
\end{array}\right.
\end{aligned}
$$

where $H \in \mathbb{R}^{N \times N}$ and $V \in \mathbb{R}^{N \times N}$ are matrix representations of, respectively, the horizontal and vertical first-order discrete differences, and where the total variation reads, for every $\eta=\left(\eta^{(n)}\right)_{1 \leq n \leq N} \in \mathbb{R}^{N}$ and $\zeta=\left(\zeta^{(n)}\right)_{1 \leq n \leq N} \in \mathbb{R}^{N}$,

$\rho_{T V}:\left(\left(\eta^{(n)}\right)_{1 \leq n \leq N},\left(\left(\zeta^{(n)}\right)_{1 \leq n \leq N}\right) \mapsto \sum_{n=1}^{N} \sqrt{\left|\eta^{(n)}\right|^{2}+\left|\zeta^{(n)}\right|^{2}}\right.$.

The functions involved in (7) are convex, lower semi-continuous, and proper. $H$ and $V$ are diagonalizable in the Fourier domain and the proximity operator of each function has a closed form. It results that the proximal algorithms PPXA+ [31] can be used to efficiently find the minimum of (7).

Estimation of $\left(v_{q}\right)_{1 \leq q \leq Q} \quad$ The a priori choice of $\left(v_{q}\right)_{1 \leq q \leq Q}$ is likely to strongly impact the estimated $\left(\theta_{q}\right)_{1 \leq q \leq Q}$. Here, we propose to extract this values from a denoised estimator of $f$ such that

$$
f^{*}=\arg \min _{g}\|f-g\|^{2}+\lambda \rho_{\mathrm{TV}}(H g, V g)
$$

where $\lambda>0$ denotes a regularization parameter, that impacts the quality of the denoised estimate. Section 4 further details the estimation of the $\left(v_{q}\right)_{1 \leq q \leq Q}$ from $f^{*}$.

\section{RESULTS}

Numerical simulations. The potential and performance of the combined estimation and segmentation procedures described in Sections 2 and 3 above are illustrated and assessed by application to independent realizations of synthetic images. They are produced numerically according to a 2D multifractional Brownian field model $[14,18]$, whose definition has been slightly modified here to ensure an homogeneous variance across the image:

$$
X(\underline{x})=C(\underline{x}) \int_{\mathbb{R}^{2}} \frac{e^{\imath \underline{x} \underline{\xi}-1}}{|\underline{\xi}|^{h(\underline{x})+\frac{1}{2}}} d W(\underline{\xi}),
$$

where $d W(\xi)$ is $2 \mathrm{D}$ Gaussian white noise and $h(\underline{x})$ denotes the prescribed Hölder exponent function. The normalizing factor $C(\underline{x})$ ensures that the local variance of $X$ does not depend on the location $\underline{x}$. This model is chosen here for convenience in synthesis. Note that the proposed estimation and segmentation procedures do not rely at all on any knowledge of the model. A sample field of such processes is shown in Fig. 1-b. Analysis is conducted using a standard 2D DWT with orthonomal tensor product Daubechies mother wavelets with $N_{\psi}=2$ vanishing moments. Regularity is estimated using the scaling range $\left(j_{1}, j_{2}\right)=(1,3)$.

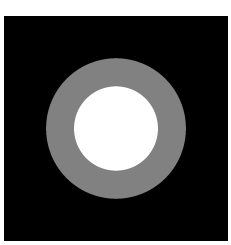

(a)

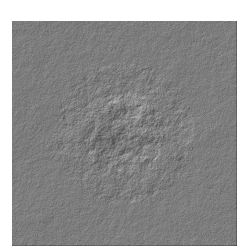

(b)

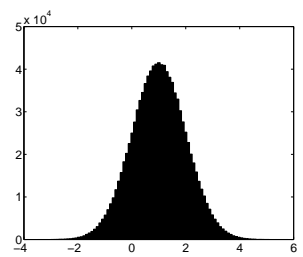

(c)
Fig. 1. Synthetic data. (a) Piecewise constant Hölder exponent mask $\left(H_{1}, H_{2}, H_{3}\right)=(0.25,0.50,0.75)$, (b) Sample field, and (c) Corresponding histogram of the pixel values.

Estimation. To compare estimation performance obtained with wavelet leaders against those produced with wavelet coefficients, the estimation procedure described in Section 2 is applied to 100 independent copies, of size $N \times N$, with $N=1024$ and with uniform Hölder exponent: $h(\underline{x}) \equiv H$. Table 1 reports estimation performance for different values of $H$ and clearly indicates that leader-based estimates of $h$ systematically outperform wavelet coefficient based ones, with significant decrease in variances at the price of increased biases, overall yielding substantially decreased mean-square errors.

\begin{tabular}{|r||r|r||r|r||r|r||}
\hline \multicolumn{1}{|c||}{$H=$} & \multicolumn{2}{c||}{0.25} & \multicolumn{2}{c||}{0.50} & \multicolumn{2}{|c||}{0.75} \\
\hline \hline & WavC & WavL & WavC & WavL & WavC & WavL \\
\hline \hline Bias & -0.36 & 0.03 & -0.19 & 0.08 & -0.08 & 0.11 \\
\hline Std & 0.52 & 0.19 & 0.55 & 0.37 & 0.60 & 0.50 \\
\hline RMSE & 0.63 & 0.19 & 0.58 & 0.38 & 0.60 & 0.52 \\
\hline min & -0.14 & 0.27 & 0.27 & 0.47 & 0.56 & 0.69 \\
\hline max & -0.09 & 0.31 & 0.37 & 0.70 & 0.77 & 1.10 \\
\hline
\end{tabular}

Table 1. Estimation Performance. Coefficients vs leaders.

Segmentation. Synthetic data are produced according to the model in (9) with piecewise constant Hölder exponent function $h(\underline{x})=H_{q}$ for Region $q$. Each region is thus regarded as a different texture and the goal is to achieve texture segmentation of the image. As an instructive example, we use images consisting of $Q=3$ different regions with piecewise constant Hölder exponents on concentric disks, as shown in Fig. 1-a. The three different textures can clearly not be identified from the histogram of the image pixel values shown in Fig. 1-c. In simulations, $N \times N=512 \times 512$.

The estimation procedures of Section 2 are applied to such images, yielding local estimates of the Hölder exponents which are plotted as images and histograms in Fig. 2-a (wavelet coefficients) and in Fig. 3-a (wavelet leaders). Despite a visual perception of 3 different regions for the wavelet coefficient based estimates $\widehat{h}_{d}$, the corresponding histogram (Fig. 2-b) is unimodal and fails to reveal the existence of three constant- $h$ regions in the image, a direct 


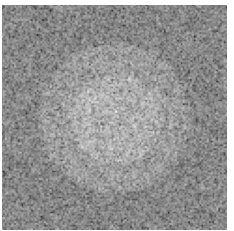

(a)

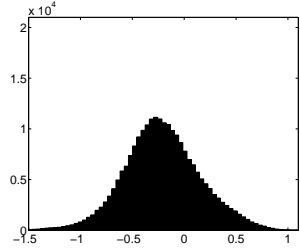

(b)

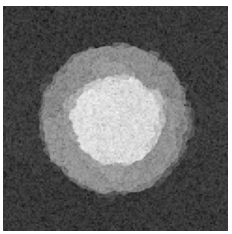

(c)

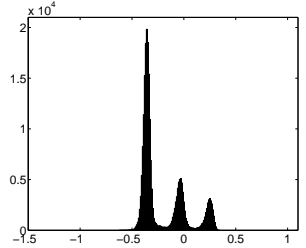

(d)

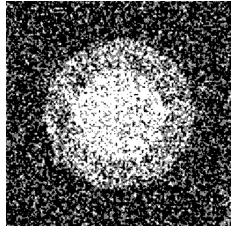

(e)

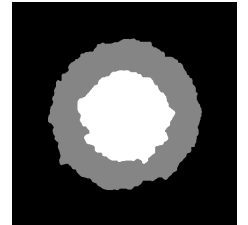

(f)

Fig. 2. Wavelet coefficient segmentation. (a) Estimate $\widehat{h}_{d}$, (b) Histogram of $\widehat{h}_{d}$, (c) $\widehat{h}_{d}^{*}$, (d) Histogram of $\widehat{h}_{d}^{*}$, (e) Segmentation based on thresholding of $\widehat{h}_{d}^{*}$, and (f) Labeling function $u$ obtained from proximal segmentation with $f=\widehat{h}_{d}$.

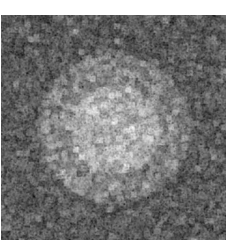

(a)

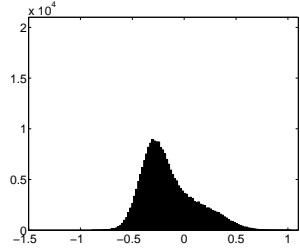

(b)

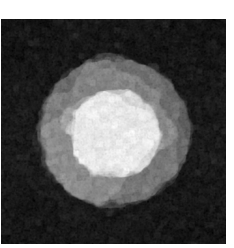

(c)

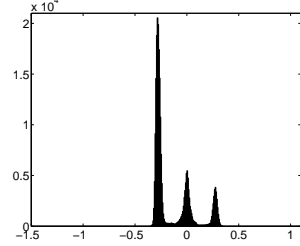

(d)

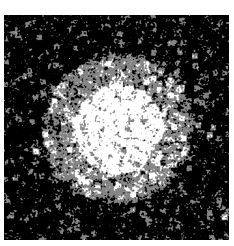

(e)

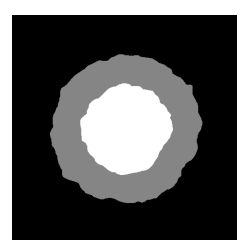

(f)

Fig. 3. Wavelet leaders segmentation. (a) Estimate $\widehat{h}_{L}$, (b) Histogram of $\widehat{h}_{L}$, (c) $\widehat{h}_{L}^{*}$, (d) Histogram of $\widehat{h}_{L}^{*}$, (e) Segmentation based on thresholding of $\widehat{h}_{L}^{*}$, and (f) Labeling function $u$ obtained from proximal segmentation with $f=\widehat{h}_{L}$.

consequence of the very large variance of the estimate $\widehat{h}_{d}$. Despite smaller variance, resulting in a non unimodal histogram (Fig. 3-b), the leader-based estimates $\widehat{h}_{L}$ (Fig. 3-a) do not clearly reveal the three constant- $h$ regions either.

The denoising procedure described in Section 3 (cf. (8)), applied to both $\widehat{h}_{d}(\underline{x})$ and $\widehat{h}_{L}(\underline{x})$, yields estimates $\widehat{h}_{d}^{*}(\underline{x})$ and $\widehat{h}_{L}^{*}(\underline{x})$ (resp. Fig. 2-c and Fig. 3-c), whose histograms, shown in Fig. 2-d and Fig. 3-d respectively, clearly display 3 modes. Segmentation based on a direct thresholding of such histograms however yields poor results as illustrated in Fig. 2-e and Fig. 3-e and Table 2. Instead, the denoising procedure is used to initialize the proximal based segmentation: The number of regions $Q$ is set to the number of local maxima observed in the denoised estimates $\widehat{h}_{d}^{*}(\underline{x})$ and $\widehat{h}_{L}^{*}(\underline{x})$, here $Q=3$; The target mean intensity values $v_{q}, q=1,2,3$ are set to values corresponding to the positions of the local maxima of the histograms of estimates $\widehat{h}_{d}^{*}$ and $\widehat{h}_{L}^{*}$.

The proximal tool based segmentation procedure described in Section 3 is now applied to both $\widehat{h}_{d}(\underline{x})$ and $\widehat{h}_{L}(\underline{x})$, initialized as described above. Results are compared in Fig. 2-f and Fig. 3-f, (for one single image) and quantified in Table 2 (averaged across 100 independent copies). This shows that the number of mis-classified pixels is systematically smaller when segmentation is based on $\widehat{h}_{L}(\underline{x})$. Differences between wavelet coefficient and leader based performance may appear small, this is however only a consequence of the chosen simple geometry for the piece-wise constant regions. Indeed, quantifying the number of mis-classified pixels within narrow strips comprising the borders between the piecewise constant- $h$ regions shows that segmentation based on $\widehat{h}_{L}(\underline{x})$ significantly outperforms that based on $\widehat{h}_{d}(\underline{x})$, a direct consequence of the better localization and lower variance of the $\widehat{h}_{L}(\underline{x})$. Wavelet leader based segmentation thus provides far better border delimitation, a crucial issue in biomedical texture segmentation, for instance. Our codes were developed in MATLAB R2011b and the segmentation of a $512 \times 512$ image with $Q=3$ labels requires only 5 minutes on an $2.4 \mathrm{GHz}$ Quad-Core Intel Xeon and 6 GB of RAM.

\begin{tabular}{|r||r|r||r|r||}
\hline $\begin{array}{r}\text { \% of miss- } \\
\text { classified pixels }\end{array}$ & \multicolumn{2}{c||}{$\begin{array}{c}\text { entire } \\
\text { image }\end{array}$} & \multicolumn{2}{c||}{$\begin{array}{c}\text { around borders } \\
\left(\sim 2.10^{4} \text { pixels }\right)\end{array}$} \\
\hline \hline & $\widehat{h}_{d}$ & $\widehat{h}_{L}$ & $\widehat{h}_{d}$ & $\widehat{h}_{L}$ \\
\hline \hline BasicThresh & 33.3 & 22.1 & 93.6 & 62.2 \\
\hline ProxSeg & 3.4 & 3.0 & 9.4 & 8.6 \\
\hline
\end{tabular}

Table 2. Segmentation Performance. Coefficients vs. leaders

\section{CONCLUSIONS AND PERSPECTIVES}

We have proposed, to the best of our knowledge, the first fully operational texture segmentation procedure based on texture local regularity. The segmentation procedure is designed for the class of piece-wise constant regularity images. It relies on combining improved regularity estimates based on wavelet leaders with the proximal solution to the minimization of the convex criterion underlying the segmentation problem. The proximal solution further relies on a total variation and proximal based initialization. The procedure is illustrated here using realizations of stochastic Gaussian model process with prescribed region-wise constant local regularity. The segmentation procedure does, however, not rely on any a priori model knowledge besides that of data belonging to the class of piece-wise constant regularity images.

The performance of the proposed procedure for different region geometry, selection of scaling range, and sensitivity to local regularity differences are currently being systematically quantified. Comparisons against alternative segmentation features, such as local entropy, will be conducted. Comparisons of the robust convex optimization based segmentation achieved here against recent classification techniques, such as the one proposed in $[28,3]$ will also be made. Moreover, extensions of the procedure to the class of piecewise smooth local regularity images are currently under study. Preliminary analysis of real biomedical textures yields promising results and will be further investigated. 


\section{REFERENCES}

[1] R. M. Haralick, "Statistical and structural approaches to texture," Proc. IEEE, vol. 67, no. 5, pp. 786-804, May 1979.

[2] A. Laine and J. Fan, "Representations for texture segmentation," IEEE Trans. Image Process., vol. 5, no. 5, pp. 771-780, May 1996.

[3] M. Jung, G. Peyré, and L. D. Cohen, "Texture segmentation via non-local non-parametric active contours," in International Conference on Energy Minimization Methods in Computer Vision and Pattern Recognition, Saint Petersburg, Russia, Jul. 2527, 2011, vol. 6819/2011, pp. 74-88.

[4] M. Unser, "Texture classification and segmentation using wavelet frames," IEEE Trans. Image Process., vol. 4, no. 11, pp. 1549-1560, Nov. 1995.

[5] L. M. Kaplan, "Extended fractal analysis for texture classification and segmentation," IEEE Trans. Image Process., vol. 8 , pp. 142-150, 1999.

[6] C.L. Benhamou, S. Poupon, E. Lespessailles, S. Loiseau, R. Jennane, V. Siroux, W. J. Ohley, and L. Pothuaud, "Fractal analysis of radiographic trabecular bone texture and bone mineral density: two complementary parameters related to osteoporotic fractures," J. Bone Miner. Res., vol. 16, no. 4, pp. 697-704, 2001.

[7] P. Kestener, J. Lina, P. Saint-Jean, and A. Arneodo, "Waveletbased multifractal formalism to assist in diagnosis in digitized mammograms," Image Analysis and Stereology, vol. 20, no. 3, pp. 169-175, 2004.

[8] R. Lopes and N. Betrouni, "Fractal and multifractal analysis: A review," Medical Image Analysis, vol. 13, pp. 634-649, 2009.

[9] N. Mallick, P. P. Cortet, S. Santucci, S. G. Roux, and L. Vanel, "Discrepancy between subcritical and fast rupture roughness: A cumulant analysis," Phys. Rev. Lett., vol. 98, pp. 255502, 2007.

[10] S. G. Roux, A. Arneodo, and N. Decoster, "A wavelet-based method for multifractal image analysis. III. Applications to high-resolution satellite images of cloud structure," Eur. Phys. J. B, vol. 15, no. 4, pp. 765-786, 2000.

[11] J. Lévy Véhel, P. Mignot, and J. Berroir, "Multifractals, texture and image analysis," in Proc. of Comp. Vis. Pattern Recognition, Jun. 15-18, 1992, pp. 661-664.

[12] B. Pesquet-Popescu and J. Lévy Vehel, "Stochastic fractal models for image processing," IEEE Signal Process. Mag., vol. 19 , no. 5, pp. 48-62, Sep. 2002.

[13] S. Jaffard, "Wavelet techniques in multifractal analysis," in Fractal Geometry and Applications: A Jubilee of Benôt Mandelbrot, M. Lapidus and M. van Frankenhuijsen Eds., Proceedings of Symposia in Pure Mathematics, M. Lapidus and M. van Frankenhuijsen, Eds. 2004, vol. 72, pp. 91-152, AMS.

[14] A. Benassi, S. Jaffard, and D. Roux, "Gaussian processes and pseudo-differential elliptic operators," Rev. mat. Iberoamericana, vol. 8, no. 1, pp. 19-89, 1997.

[15] P. Gonçalvès and P. Abry, "Multiple window and local scaling exponent estimation," in Proc. Int. Conf. Acoust. Speech Signal Process., Munich, Germany, Apr. 21-24, 1997, pp. 3433-3436.

[16] A. Benassi, S. Cohen, and J. Istas, "On roughness indices for fractional fields," Bernoulli, vol. 10, no. 2, pp. 357-373, 2004.
[17] B. Mandelbrot and J. W. van Ness, "Fractional Brownian motion, fractional noises and applications," SIAM Reviews, vol. 10, pp. 422-437, 1968.

[18] A. Ayache, S. Cohen, and J. Lévy Vehel, "The covariance structure of multifractional brownian motion, with application to long range dependence," in Proc. Int. Conf. Acoust. Speech Signal Process., Dallas, Texas, USA, Mar. 14-19, 2000, pp. 3810-3813.

[19] M. Kass, A. Witkin, and D. Terzopoulos, "Snakes: Active contour models," Int. J. Comp. Vis., vol. 1, no. 4, pp. 321-331, Jan. 1988.

[20] D. Mumford and J. Shah, "Optimal approximations by piecewise smooth functions and associated variational problems.," Comm. Pure Applied Math., vol. 42, no. 5, pp. 577-685, Jul. 1989.

[21] V. Caselles, R. Kimmel, and G. Sapiro, "Geodesic active contours," in Proc. IEEE Int. Conf. Comput. Vis., Boston, MA, USA, Jun. 20-23, 1995, pp. 694-699.

[22] S. Kichenassamy, A. Kumar, P. J. Olver, A. Tannenbaum, and A. J. Yezzi, "Gradient flows and geometric active contour models," in Proc. IEEE Int. Conf. Comput. Vis., Boston, MA, USA, Jun. 20-23, 1995, pp. 810-815.

[23] T. F. Chan and L. A. Vese, "Active contours without edges," IEEE Trans. Image Process., vol. 10, no. 2, pp. 266-277, Feb. 2001.

[24] C. Couprie, L. Grady, L. Najman, and H. Talbot, "Power watershed: A unifying graph-based optimization framework," IEEE Trans. Pattern Anal. Match. Int., vol. 33, no. 7, pp. 1384-1399, Jul. 2011.

[25] T. Chan, S. Esedoglu, and M. Nikolova, "Algorithms for finding global minimizers of image segmentation and denoising models," SIAM J. App. Math., vol. 66, no. 5, pp. 1632-1648, 2006.

[26] T. Pock, A. Chambolle, D. Cremers, and B. Horst, "A convex relaxation approach for computing minimal partitions," in Proc. of Comp. Vis. Pattern Recognition, Miami, Florida, USA, Jun. 20-25, 2009, pp. $\mathrm{x}+8$.

[27] S. Hiltunen, J.-C. Pesquet, and B. Pesquet-Popescu, "Comparison of two proximal splitting algorithms for solving multilabel disparity estimation problems," in Proc. Eur. Sig. and Image Proc. Conference, Bucharest, Romania, Aug. 27-31, 2012, pp. $\mathrm{x}+5$.

[28] K. Ni, X. Bresson, T. F. Chan, and S. Esedoglu, "Local histogram based segmentation using thewasserstein distance," Int. J. Comp. Vis., vol. 84, no. 1, pp. 97-111, 2009.

[29] S. Mallat, A wavelet tour of signal processing, Academic Press, San Diego, USA, 1997.

[30] H. Wendt, P. Abry, and S. Jaffard, "Bootstrap for empirical multifractal analysis," IEEE Signal Process. Mag., vol. 24, no. 4, pp. 38-48, Jul. 2007.

[31] J.-C. Pesquet and N. Pustelnik, "A parallel inertial proximal optimization method," Pac. J. Optim., vol. 8, no. 2, pp. 273305, Apr. 2012. 